\title{
Blackeye bean root rot diseases identified
}

\author{
Donald C. Erwin \\ Carol Frate $\square$ Douglas Munier
}

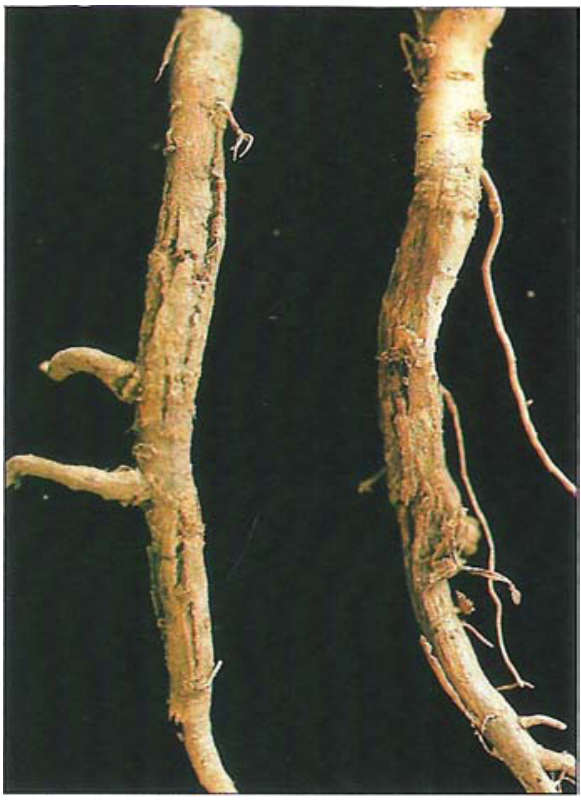

Roots of black-eye bean plants from the field showing longitudinal cracking.

\section{Premature yellowing, aging and crop loss in blackeye bean is asso- ciated with root rot diseases caused by Fusarium solani and Thielaviopsis basicola. Now a new root rot disease caused by Phytophthora drechsleri could be- come a problem in wet soil.}

With an annual value of $\$ 23$ million, blackeye bean (Vigna unguiculata) is an important crop in the San Joaquin Valley, where it covers 40,000 acres. Also known as black-eyed pea or cowpea, this highprotein legume has drought-resistant characteristics attractive when water is limited. In the past few years growers have noted premature yellowing and ag ing or senescence of lower leaves, reduction of pod set, and in some cases death of plants, even in fields in which Fusarium wilt is not a factor. When roots are removed from soil, reddish brown, blotchy lesions with diffuse margins are evident. Quite often, the lesions are shallow, but in other cases the rotting of tissue or necrosis extends into the xylem, and the bark is cracked longitudinally. Frequently; only parts of the lateral or tap roots are affected while some regions remain white and disease-free. Root lesions of varying severity can also be seen on plants with healthy tops.

The major recognized disease of blackeye bean has been Fusarium wilt caused by Fusarium oxysporum $\mathrm{f}$. sp. tracheiphilum. Fusarium wilt can be readily diagnosed by cutting the lower stem with a sharp knife and looking for a dark brown color in the xylem tissue. Root rot is not a primary symptom of this disease. In 1989 Fusarium wilt was found in $\mathbf{3 5}$ of 47 fields (74\%) that were surveyed in Kern and Tulare Counties. Although Fusarium wilt is probably more widespread than was expected, the early cutout condition was even more widespread and occurred in the absence of Fusarium wilt.

Over a 3-year period beginning in 1987, we surveyed and investigated the root diseases of blackeye bean at the UC Riverside Agricultural Experiment Station and in farmers' fields in Tulare and Kern counties. During late July, August, and early September of 1987, 1988, and 1989, plants were collected in the field, examined for symptoms and fungi were isolated from affected roots. Root rot was prevalent in over $50 \%$ of the plants.

\section{Isolations}

Isolations of associated fungi were made using standard nonselective media as well as media selective for Fusarium fungi. Roots were washed, surfacedisinfested with $10 \%$ commercial chlorine bleach, and washed again in sterilized distilled water. One-half-centimeter-square pieces were also placed on several media selective for culturing Phytophthora and Pythium species.

Isolations yielded:

Fusarium oxysporum f. sp. tracheiphilum, cause of Fusarium wilt, mainly from plants with vascular discoloration, at relatively high frequency,

- Fusarium solani and Thielaviopsis basicola, both capable of causing root and hypocotyl rot,

- two Pythium spp., tentatively identified as $P$. aphanidernatum and $P$. acanthicum, known to cause seedling disease, lateral root rot, and Pythium wilt,

- Rhizoctonia solani, cause of root and stem canker,

Macrophomina phaseolina, which causes ashy stem blight, and,

in two different fields in different years, Phytophthora drechsleri, which causes root rot of several crops.

The most frequently isolated fungus was F. oxysporum f. sp. tracheiphilum, followed by $F$. solani and $T$. basicola, at about equal frequency. Two $P$ ythium species, $P$.

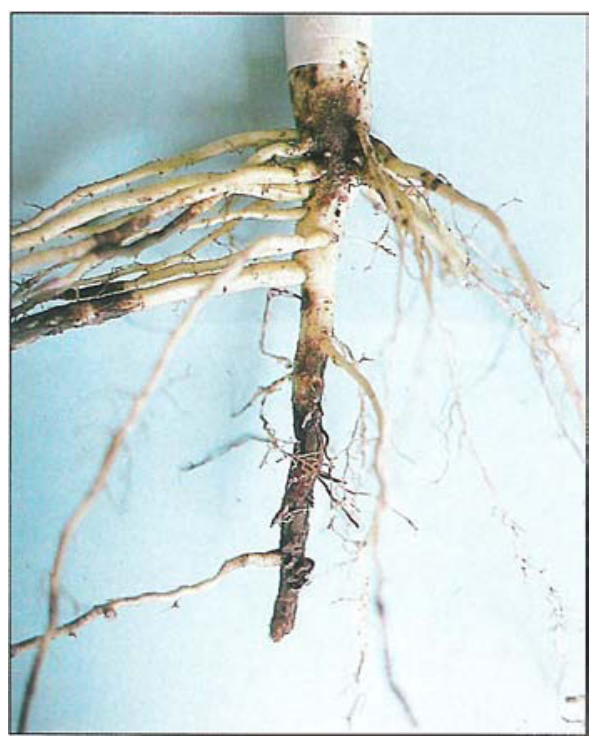

Roots of black-eye bean plant from the field with rotted lateral tap roots.

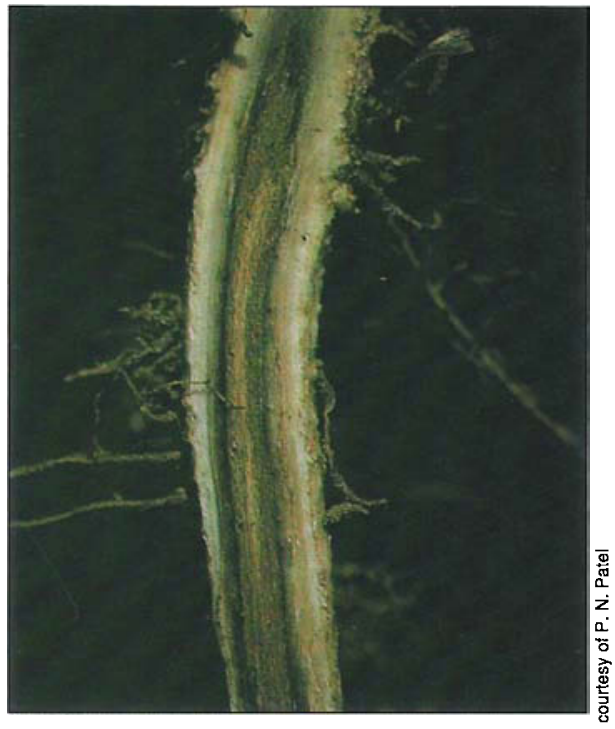

Cowpea plant with the vascular necrosis wilt symptom caused by Fusarium oxysporum $\mathrm{f}$. $\mathrm{sp}$. tracheiphilum, cause of Fusarium wilt . 

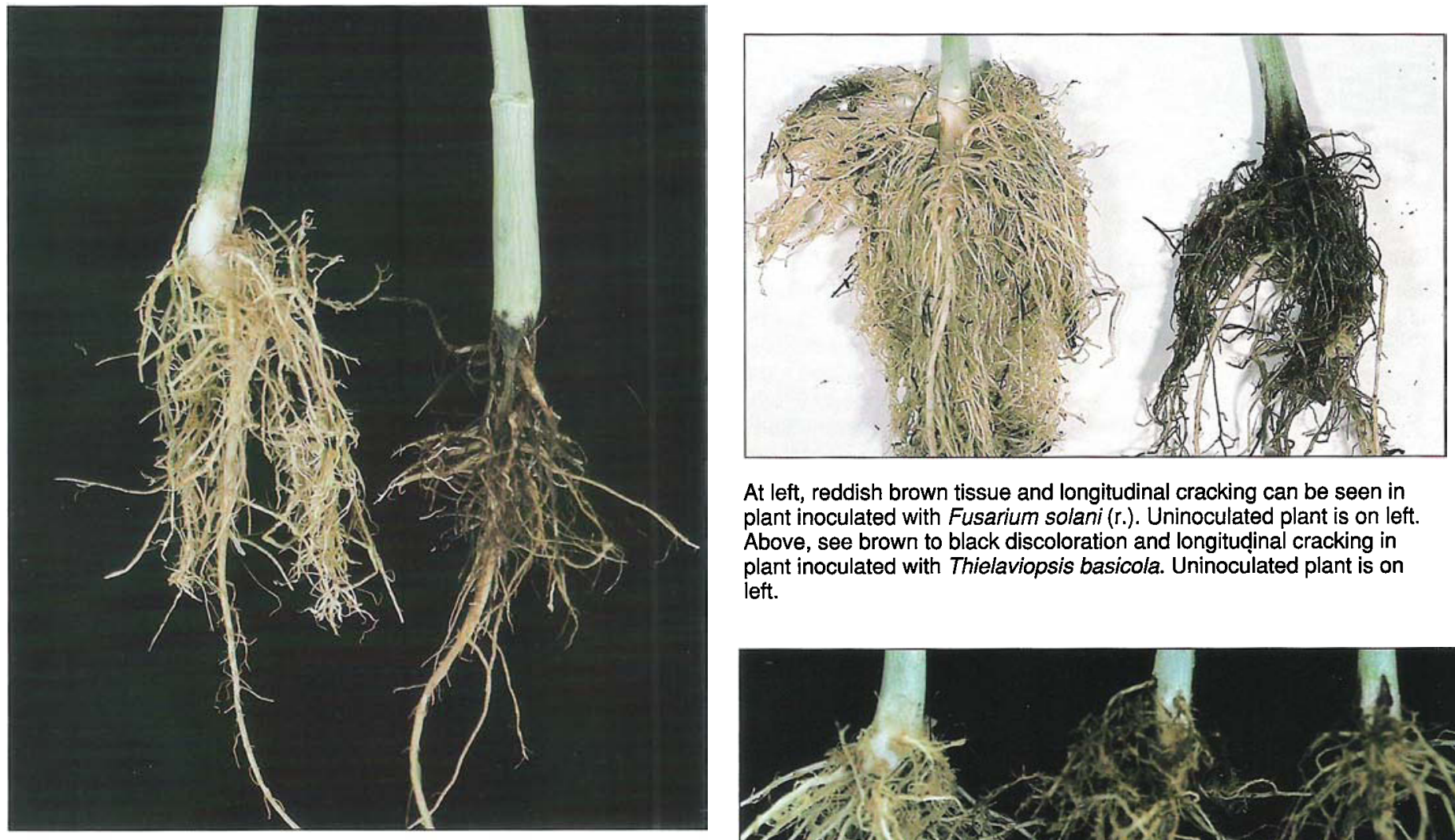

At left, reddish brown tissue and longitudinal cracking can be seen in plant inoculated with Fusarium solani (r.). Uninoculated plant is on left. Above, see brown to black discoloration and longitudinal cracking in plant inoculated with Thielaviopsis basicola. Uninoculated plant is on left.

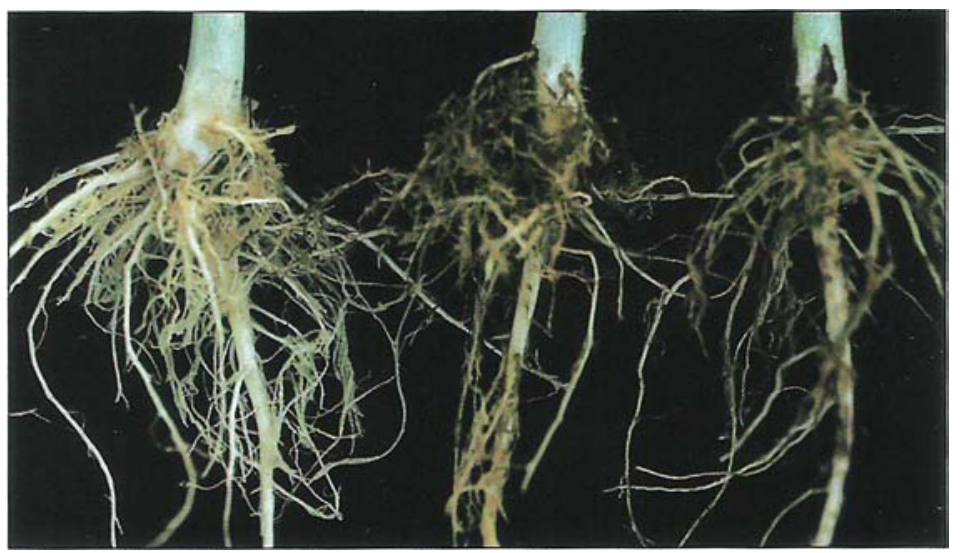

At left, note the round sunken canker of a plant inoculated with Rhizoctonia solani. Uninoculated plant is on left. Above, note the brown discoloration of the rotted tissue in two plants inoculated with Phytophthora drechsleri. Plant on the left was not inoculated.

aphanidernatum and $P$. acanthicum, were isolated frequently; Rhizoctonia solani, infrequently; Macrophomina phaseolina, infrequently and mainly in the latter part of the growing season; and in two different fields, each in different years, $P$. drechsleri, infrequently. The latter fungus has not been reported previously on blackeye bean.

\section{Pathogenicity tests}

The isolated fungi were cultured in the laboratory for use as inoculum to test their ability to cause disease symptoms.

Blackeye bean plants (cv. California Blackeye 5) were grown two per plastic pot containing UC steamed potting mix (50\% fine sand and $50 \%$ peat moss) and fertilized with a balanced nutrient solution and one application of a slow-release fer- tilizer, Osmocote (18-6-12, $20 \mathrm{~cm}^{3} /$ pot.) Plants were grown in a glasshouse at temperatures ranging from $70^{\circ} \mathrm{F}$ night to a maximum of $86^{\circ} \mathrm{F}$ in daytime.

When plants were 30 days old we placed inoculum in a hole 1 to 2 inches deep near the root system of each plant. Control plants were not inoculated. After inoculation with the water mold $P$. drechsleri, plants were flooded with water and kept wet by maintaining full saucers of water under each pot for 3 out of 7 days per week during two cycles. Symptoms were recorded after 30 days and reisolations made to confirm the presence of the isolate used for inoculation.

\section{Results}

Plants inoculated with Fusarium oxysporum f. sp. tracheiphilum isolates showed lower leaf yellowing and browning of woody tissue, but little or no tap root or lateral root rot. This indicated that the root rot symptoms seen in the field were not caused by this fungus.

Most of the $F$. solani isolates collected at UC Riverside and in Tulare and Kern counties were highly infectious. Isolate 1337-4-2 reduced plant dry weight to $51 \mathrm{~g}$ from $70 \mathrm{~g}$ in the control. At least 10 other isolates from Tulare, Kern and Riverside Counties were tested with similar results. One isolate, 1328-3-1, caused severe root rot, but did not affect growth (table 1).

When plants inoculated with $F$. solani were removed from the soil, the upper tap root was orange to brown. This root symptom was similar to the reddish blotchy lesions seen in the field and could be readily differentiated from the darker brown to 

caused by $T$. basicola.

Plants inoculated with $T$. basicola isoQuite often, the cortical tissue was cracked longitudinally. Dry weight of a two-plant replicate was reduced from $70 \mathrm{~g}$ in the control to $35 \mathrm{~g}$ for one isolate and $43 \mathrm{~g}$ for another (table 1). An isolate from cotton was also highly virulent on black-eye bean (data not shown).

Macrophomina phaseolina is a common pathogen, which often causes ashy stem blight following stress, but in our inoculations it had little or no effect on growth or root rot under the conditions tested (table 1). This may have been due to the lack of heat or drought stress imposed on the plants in the greenhouse experiments. Field experience indicates that both types of stress predispose blackeye and common

Rhizoctonia solani, which is a soil inhabitant common to all agricultural soils, was isolated less frequently than $F$. solani or $T$. basicola. The fungus caused red cankers about $1 / 2$ inch in diameter with distinct margins on the upper tap roots of inoculated plants. The sunken and sharply defined margins of the canker differentiated this from other symptoms associated with Fusarium cortical rot and Thielaviopsis black root rot. Two $R$. solani isolates tested caused root cankers but not foliar symptoms. Based on the symptoms seen in the field and on those produced in the greenhouse inoculations, we doubt that $R$ : solani is more than a minor cause of premature senescence.

Phytophthora drechsleri, a water mold also known to be a pathogen of safflower and sugar beet, was isolated for the first black discoloration of cortical tissues lates had severe black root rot symptoms at the crown level and on the main roots. bean to ashy stem blight.

time in 1988 and again in 1989 in Tulare County. Inoculation with this fungus resulted in extensive and severe tap and lateral root rot. The color of the rotted tissue was tan to brown.

The severity of root rot caused by an isolate of $P$. drechsleri from blackeye and another from sugar beet was high. Both isolates caused foliar chlorosis and wilting. In one experiment $P$. drechsleri reduced the height of the control from 47 to 16 inches. This is the first report in the literature of a root rot of blackeye bean caused by $P$. drechsleri.

The Pythium isolates caused a lateral root rot that often extended into the main tap root, but the effect was not as drastic as that caused by P.drechsleri. These Pythium isolates may be potentially important and would most likely be a problem if irrigation were excessive. We noted that these isolates were also capable of causing "Pythium wilt," a field disease characterized by a collapsed area of dying tissue on the stem. This reddish-bordered region is caused by necrosis (tissue death) extending upward through the pith from the roots. In our experience, the incidence of this disease in the field has not been high.

\section{Discussion and conclusions}

We conclude that the early cutout or premature senescence seen in a large proportion of blackeye bean fields in the San Joaquin Valley was caused primarily by Fusarium solani f. sp. phaseoli. F. solani caused root symptoms similar to those seen on roots in the field. This fungus, identified by Shirley Nash Smith of the Snyder and Hansen Institute in Alameda as F. solani f. sp. phaseoli, caused disease in common bean (Phaseolus vulgaris), but not in pea (Pisum satioum) (data not shown).

TABLE 1. Effect of inoculation of California Blackeye 5 with several fungi isolated from diseased roots

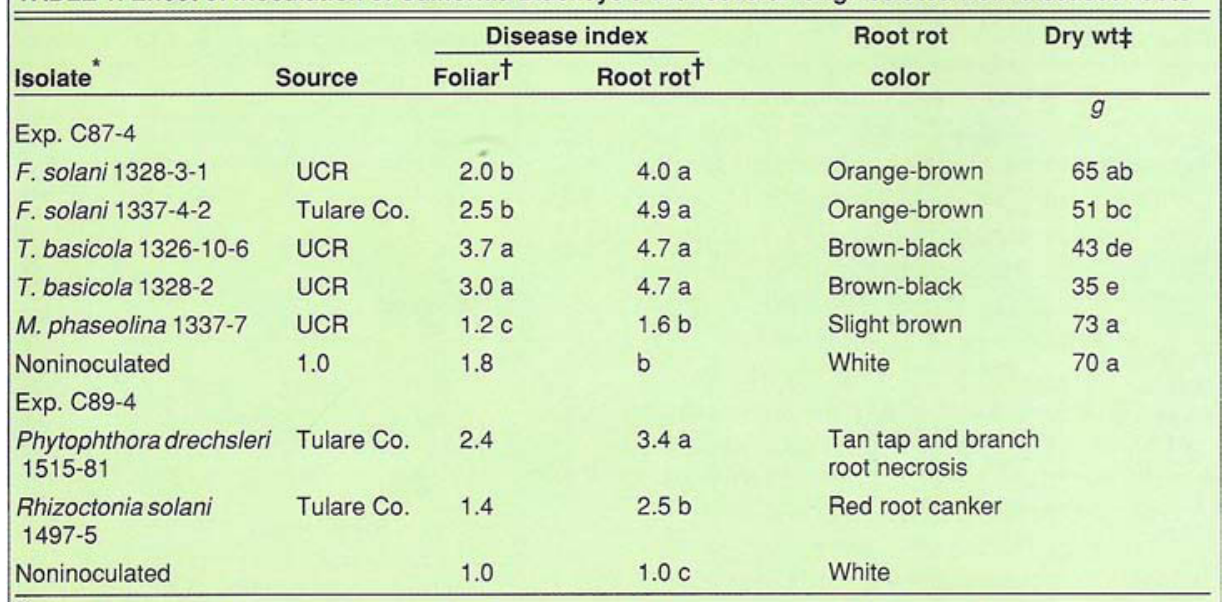

F. = Fusarium. ; M. = Macrophomina; $T$. = Thielaviopsis; UCR = University of California Riverside.

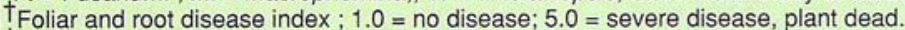

¥ Dry weight of two plants per replicate pot. Data followed by the same letter do not differ significantly $(P=0.01)$ by Duncan's multiple range test.
Fusarium cortical rot of both blackeye bean and common bean is not new, but there has been relatively little research conducted on the disease on blackeye bean. Most Fusarium solani propagules are considered long-lived in soil and capable of building large populations when in the presence of a susceptible host .

Thielaviopsis basicola, which was also isolated from some fields, causes a black root rot and is widespread in the central valleys. This fungus may also be an important component of the root rot complex, but since the black root symptoms were not seen frequently, we do not think it is as important as Fusarium cortical rot. It also causes black root of cotton, a serious postemergence disease which may cause up to $50 \%$ yield loss in some fields according to research by Kater Hake and others in Kern County (see the 1985 California Cotton Progress Report). This disease becomes more severe in direct relation to the inoculum level in the soil. Since an isolate from cotton was equally as pathogenic to blackeye bean as isolates from blackeye bean, its role in rotation of blackeye bean with cotton should be considered.

Other experiments at Prosser, Washington showed that common bean plants grown on compacted soil are predisposed to severe root disease caused by $F$. solani $\mathrm{f}$. sp. phaseoli and that ripping soils before planting reduces disease severity. In some cases, farm advisors have noted that such treatments alleviate the effects of early cutout of blackeye. In some fields in Kern County, we have seen the tap root of blackeye plants diverted to horizontal growth by a hard pan layer in soil. Maintenance of optimal growing conditions might alleviate the effects of root rot pathogens.

From our observations, it appears highly likely that early cutout is increased in severity by certain cultural practices that impose stress on the plants. Some of these might include excessive drought or irrigation. Rotations to resistant grass-type crops, like corn, milo, or barley, would help to reduce the inoculum concentration in soil; however, since both $F$. solani and $T$. basicola produce long-lived spores, short rotations might not be effective. We are currently screening plants to find a source of resistance in blackeye bean germplasm.

D. C. Erwin is professor, R. A. Khan is Staff Research Associate, and C. Ver Linden is a former graduate student, Pest Management, UC Riverside; Carole Frate is Farm Advisor, Tulare County, and Douglas Munier is Farm Advisor, Kern County. The authors gratefully acknowledge financial support from the California Dry Bean Advisory Board. 